\title{
Research on Visualization of Marine Data
}

\author{
Li Haitao 1, a), Guan Yu 2, b) \\ ${ }^{1}$ School of Information Science and Technology, Qingdao University of Science and Technology, \\ Qingdao ShanDong 266000, China. \\ ${ }^{2}$ School of Information Science and Technology, Qingdao University of Science and Technology, \\ Qingdao ShanDong 266000, China. \\ a)32891741@qq.com, ${ }^{\text {b) } 116707600 @ q q . c o m}$
}

Keywords: Visualization, Marine data

\begin{abstract}
According to the marine data types are complex, the format is different, in this paper, the relevant visualization techniques and representative methods are studied, and the visualization of marine data is compared and analyzed. The visualization of marine data spatial information based on GIS technology is discussed and the process of spatial information visualization of marine data is designed.
\end{abstract}

\section{OVERVIEW OF VISUALIZATION TECHNOLOGY}

The objective reality constitutes the people in the brain can accept the image of the process and methods, or is an intuitive expression of something that cannot be directly aware of what we call visualization. Through the visualization technology, can easily convert data and information into people can intuitively cognitive color, table, multi-dimensional graphics, images, animation and other forms. Through this technology can make the contents of the data more clearly and vividly displayed on the screen. Visualization research can be summarized as the following two aspects: First, the visualization technology is the vertical technical level, that is, performance and display of research data. In short, is to study a tool that allows the data into a visualized image, in order to facilitate understanding. The second is the horizontal application of visual technology level, will be applied to science and engineering visualization of the various disciplines. The study of visualization technology involves computer-aided design, computer vision, computer graphics and image processing and other related fields ${ }^{[1]}$.

The definition, the areas covered and the direction of recent and long-term research of scientific computing visualization, were formally presented in 1987 at a symposium held by the National Science Foundation of the United States. The use of computer graphics and image processing technology, the process of generating data and calculation results are converted to graphics and images displayed, and interactive processing theory, methods and techniques ${ }^{[2]}$, known as scientific computing visualization. The use of graphical way to massive data contained in the phenomenon and the law shown, to promote people's insight and understanding of the data to a comprehensive and essential grasp of the basic characteristics of the data to facilitate the rapid and vivid transmission and reception of them ${ }^{[3]}$. The application fields of Visualization in Scientific Computing are very extensive, covering almost all fields of natural science and engineering technology. The main application fields include medical CT, geological exploration, Physical Ocean and so on. Visualization is an effective way to study the law of data analysis by studying visualization technology and developing visualization application system to convert a large number of abstract data into graphic images which can be directly perceived and perceived ${ }^{[4]}$.

\section{VISUAL EXPRESSION}

The visualization of marine data can be divided into three types according to different standards and different rules, to be different types of visualization display.

\subsection{Static and dynamic data visualization}

According to the static or dynamic characteristics of different marine data, the visualization of 
the rules or phenomena can be divided into static data visualization and dynamic data visualization.

\subsubsection{Static data visualization}

For the statistical analysis of some static characteristics of marine data, statistical charts can be used to express the way. In the field of marine information, the chart is the basic means of data visualization, and the basic chart - histogram, curve chart, pie chart is most commonly used. The basic chart, which is often used to facilitate understanding of large amounts of data, as well as the relationships between the data, the basic chart of the marine is used to present the trend of marine information data. While similar to the wind direction rose charts, radar charts and other quantitative statistical charts, are often based on marine geographic information, presents a specific geographical chart background, not only can express the visual time and space distribution characteristics of the sea, but also highlights the fixed time segment of the marine data between the differences and contrast ${ }^{[1]}$.
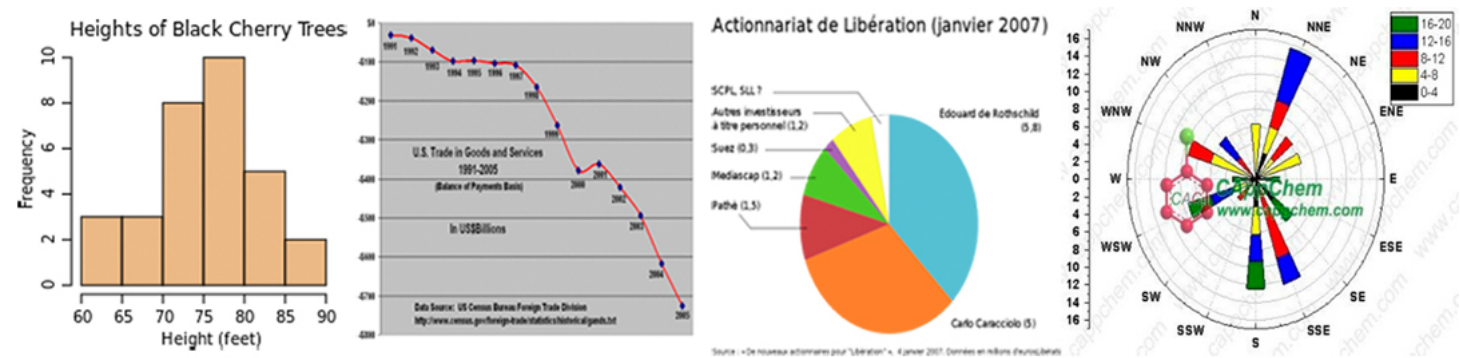

FIGURE 1. Static Data Visualization

\subsubsection{Dynamic data visualization}

Dynamic data usually refers to data that changes over time and is often used to describe the dynamics of complex marine data in the visual field. In this dynamic process of change, is in accordance with the time of the order of change. Due to color or flag changes, and thus reflect the different changes in the form of the process, according to time series or map sequence in order to display. By means of the above-mentioned manner, the process of changing the phenomenon of marine data characteristic with time can be demonstrated concretely.
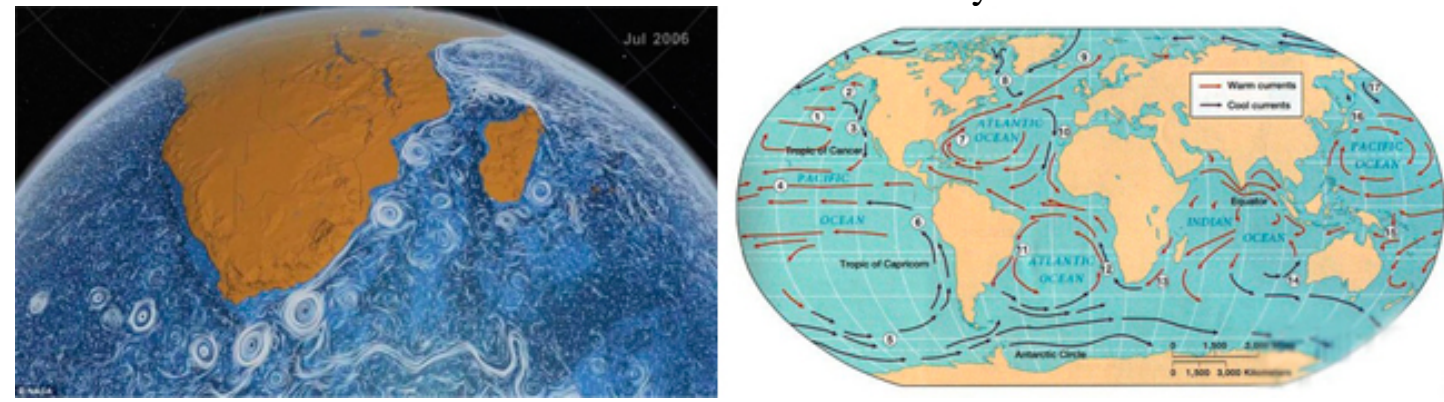

FIGURE 2. Dynamic Data Visualization

\section{2 $\mathrm{N}$-dimensional scalar data visualization}

According to the characteristics of information dimension (space-time latitude, elements latitude etc.) of marine data expression, the graph can be divided into one-dimensional scalar data visualization, two-dimensional scalar data visualization and three-dimensional scalar data visualization.

\subsubsection{One-Dimensional scalar data visualization}

One-dimensional space scalar data generally refers to the data collected along a certain path in the space, such as the temperature or salinity collected by sea station in seawater; One-dimensional time scalar data in general refers to the value of a scalar change over time, such as the sea station in seawater at the same depth every 1 hour to read the nutrient concentration.

A set of one-dimensional scalar data points $\mathrm{x} 1$, and a y-value $\mathrm{F}(\mathrm{x} 1)$ sampled at each point. From the function $\mathrm{F}(\mathrm{x} 1)$ sampling data, the graphical visualization methods including graphs, bar graphs, pie charts and other visualization.

(1) Fold (curve) line diagram

Fold (curve) line graph is mainly used to show the development and change of the law or trend of things at different times. Draw a graph of the fold (curve) from the given data point to visualize 
the function F (X1). Among them, the superposition of the fold (curve) line shows the same chart, respectively drawing two or more fold (curve) line, and according to the different drawing techniques (color, thickness, etc.) to distinguish the lines. Therefore, the superposition of the fold (curve) line can not only reduce the redundancy of the space used to represent information, but also a good expression of the relationship between the various variables.

(2) Bar (histogram) chart

Bar (histogram) chart can be displayed horizontally or vertically, with the rectangular (column) of the length to reflect the difference in the data. It is usually applied to a two-dimensional data set. The bar chart uses its length and the histogram uses its height to represent the size of the data value. The width represents the size of the domain interval corresponding to the data value, and color can be used to distinguish each column in order to change the focus on the abscissa trend.

(3) Pie chart

When each data value is the total amount of each component, the size of each pie in the circle represents the percentage of the total number of parts, the percentage of its total and certainly 1 , it is a whole. Using pie charts can be the most intuitive expression of the number of parts and the total number of changes.
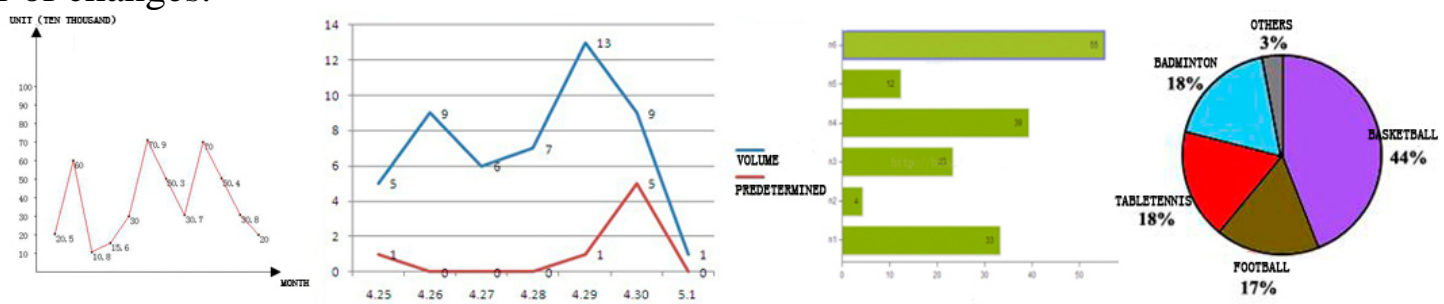

FIGURE 3. One-Dimensional Scalar Data Visualization

\subsubsection{Two-Dimensional scalar data visualization}

Two-dimensional scalar data is more common, such as medical diagnostic X-ray, ocean data profile and so on. The two-dimensional scalar data represents a visualization of the change of a certain attribute value of the marine data with the temporal and spatial characteristics.

A set of two-dimensional scalar data points $\mathrm{x} 1$, $\mathrm{x} 2$, and the $\mathrm{y}$-values $\mathrm{F}(\mathrm{x} 1, \mathrm{x} 2)$ sampled at each point. From the function $\mathrm{F}$ (X1, x2) sampling data, from the geometric point of view, the representation of the graphics are divided into contour maps, image methods and surface maps, etc..

\section{(1) Contour map}

Contour map is a graph of continuous, gradual changes in the number of features represented by lines of equal values. Contour map depends on the characteristics of the data points, select the appropriate interpolation method for processing. For different data types, can be Inverse Distance to a Power, Kriging and Natural Neighbor. Contour maps with discrete shades are used to indicate the value of the area between two adjacent contours with different colors or shades.

(2) Image method

The function values on the plane are represented by an image, and the two-dimensional area is divided into the pixels of the unit grid corresponding to the display, and the corresponding value of the function is represented by the color on each pixel.

(3) Curved surface

The surface graph can be viewed as a generalization of the linear graph method in the visualization of the one-dimensional scalar field in the two-dimensional space. It is visualized by interpolating the function value at the sampling point $(\mathrm{x} 1, \mathrm{y} 1),(\mathrm{x} 1, \mathrm{y} 2), \ldots,(\mathrm{x} 1, \mathrm{yn}),(\mathrm{x} 2, \mathrm{y} 1), \ldots$, (xm, yn) and drawing visualization with a surface. 

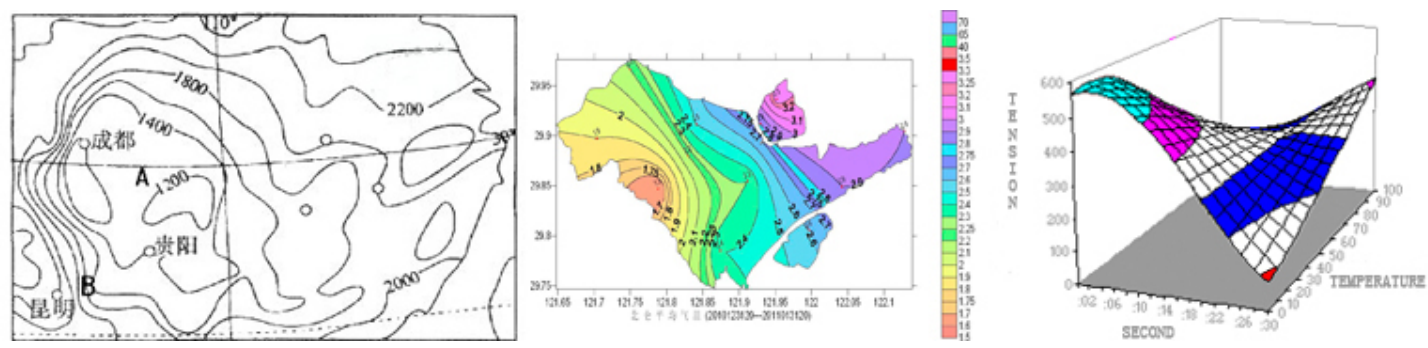

FIGURE 4. Two-Dimensional Scalar Data Visualization

\subsubsection{Three-Dimensional scalar data visualization}

Scientific research and social activities through experimental observation or simulation calculation to produce three-dimensional data, record the three-dimensional field of physical and chemical properties and its evolution. Dimensional feature of elements is represented by $(\mathrm{X}, \mathrm{Y}, \mathrm{Z})$ three-dimensional coordinates, $\mathrm{x}, \mathrm{y}$ represent the scalar data of the plane, the third dimension $\mathrm{z}$ represents the terrain height value, and the numerical value is provided by the attribute value of the marine data feature to be represented. Three-dimensional sense of space depends on the range of values across the property, the two are proportional to the relationship, when the range spans large, the corresponding expression of its quantified the stronger.

A set of three-dimensional scalar data points $\mathrm{x} 1, \mathrm{x} 2, \mathrm{x} 3$, and y values $\mathrm{F}(\mathrm{x} 1, \mathrm{x} 2, \mathrm{x} 3)$ sampled at each point, the isosurface is represented by all points $S=\{(\mathrm{x} 1, \mathrm{x} 2, \mathrm{x} 3): \mathrm{F}(\mathrm{x} 1, \mathrm{x} 2, \mathrm{x} 3)=\mathrm{Ft}\}$ curved surface. From the function $\mathrm{F}(\mathrm{x} 1, \mathrm{x} 2, \mathrm{x} 3)$ sampling data, the main methods of 3D scalar data visualization include surface rendering and volume rendering.

\section{(1) Isosurface rendering}

Constructing equivalent surface in 3D spatial data field, firstly, we construct intermediate geometric primitives, such as triangles, and then draw the surface by traditional computer graphics technology. There are many algorithms and techniques for 3D spatial data fields based on isosurface, including Marching Cubes, contour connection method and constructing the outer surface from two-dimensional contour lines.

\section{(2) Volume rendering}

Volume rendering technique is a rendering technique which directly produces corresponding 2D images from discrete 3D data fields. Unlike the isosurface method, intermediate geometric primitives are not required in this process. Volume rendering of the image is generally a translucent image, the color is generally artificial pseudo-color specified. The prerequisite of volume rendering is based on the classification of data. Each category is assigned different color and opaque value, and then depends on the relative position of the viewpoint and volume data in space to determine the final imaging effect.
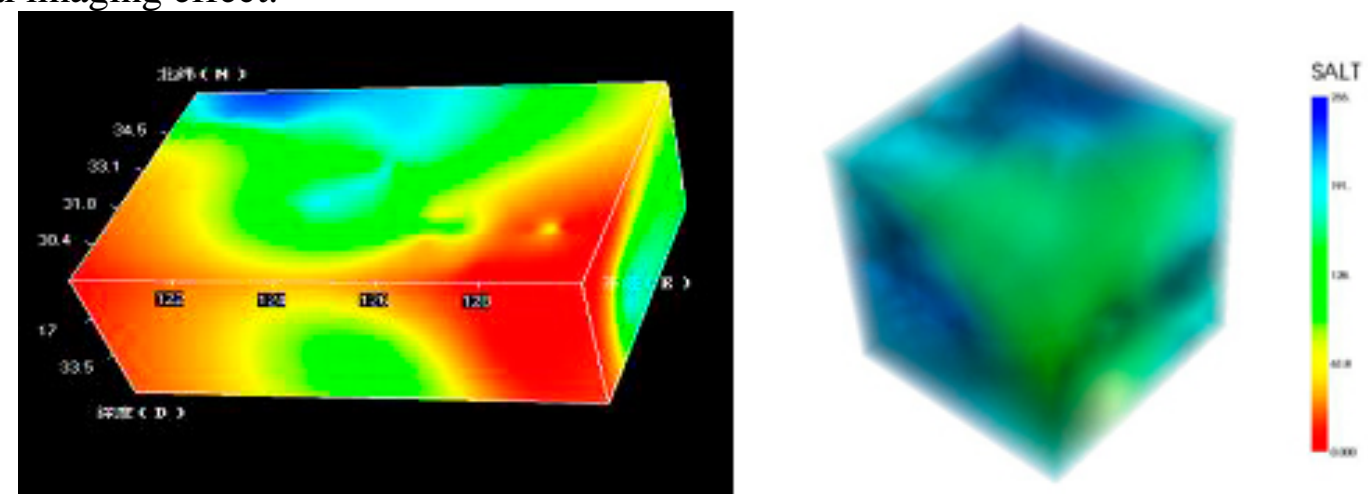

FIGURE 5. Three-Dimensional Scalar Data Visualization

\subsection{Geometric data visualization}

The data can be divided into point data, line data, surface data and volume data, which can be visualized by using geographic information visualization and marine data processing.

\subsubsection{Point data visualization}

Point data is the most common type of data in geographic data. Coordinate information in geographic space can be represented by point data, which contains longitude coordinates and 
latitude coordinates, and represents geographically discrete points that are used to identify or locate geographical entities. The simplest point data visualization is based on his coordinate information drawn on the map. In addition, the point data can be distinguished by the color, size, or other attributes, or in addition to the simple point of the other icons. As shown in FIGURE 6, the map of the voyage points in the coastal waters is displayed, which is directly mapped on the map according to the coordinates of the voyage points.

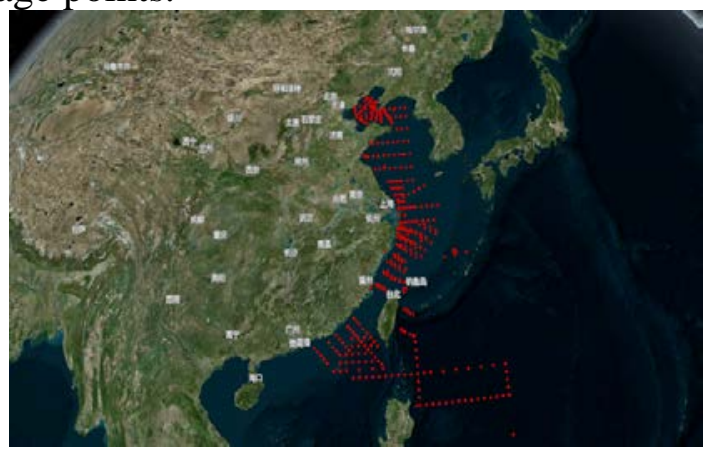

FIGURE 6. Point Data Visualization

\subsubsection{Line data visualization}

Line data in geospatial refers to a series of points connected in series. Line data can usually be used to represent the map of the marine voyage track and driving trajectory on the road , and static information on the map such as roads, railways, etc., which are composed of a series of ordered points , which contains the length information of line data. Line data visualization can be based on its two adjacent points of data, according to the order of sequential mapping in the map to complete. Visualization of the application scene is often used to show the marine voyage path trajectory, as shown in FIGURE 7.

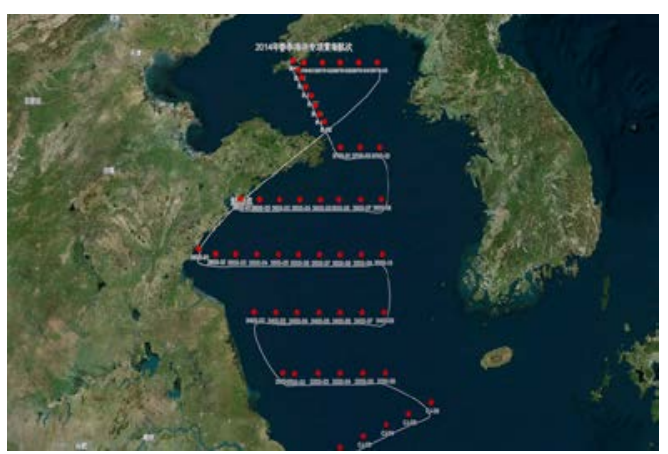

\subsubsection{Surface data visualization}

FIGURE 7. Line Data Visualization

Surface data visualization contains complex and diverse information, compared with point data and line data, the information content is more huge. The surface data is a closed space composed of a series of points, such as the surface of the building on the map, the boundary of the administrative area, and the surface of the lake, etc.. Surface data visualization can represent a region's attributes or properties, such as density, temperature, salinity, and so on. The most commonly used is to represent the size of these values by different colors, as shown in FIGURE 8, for the environmental parameter concentration profile of $\mathrm{SiO} 3$ at the depth of $0 \mathrm{~m}$ in the coastal waters.

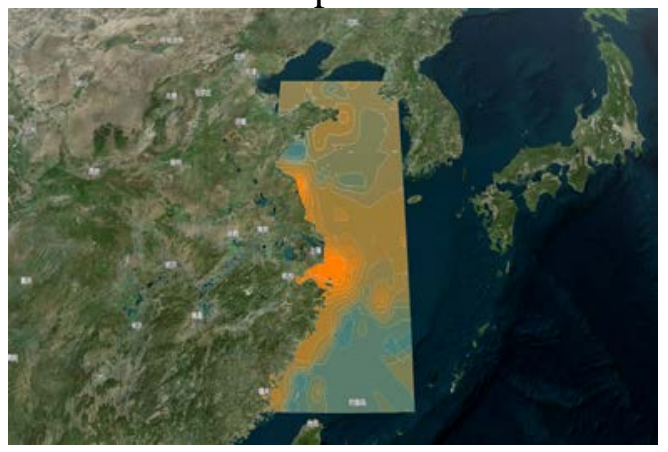

FIGURE 8. Surface Data Visualization 


\subsubsection{Volume data visualization}

Volume data is a very common type of data, recorded in a three-dimensional or multidimensional space within the discrete grid points on the value. Volume data is widely used in computer science, computer graphics, biomedicine, physical oceanography and many other fields, and has its important purpose, that can describe three-dimensional depth of information, it can be generated after the graphical effect of beautiful, or by intuitive analysis to get valuable information.

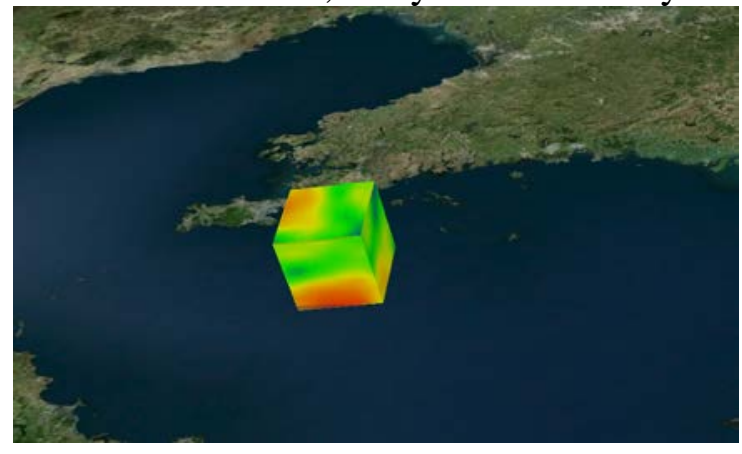

FIGURE 9. Volume Data Visualization

\section{VISUALIZATION OF MARINE DATA SPATIAL INFORMATION BASED ON GIS}

On the one hand, the use of visualization technology can be further simplified for human cognitive process of complex entities and objects, on the other hand, in some specific geographical space or closely related features and phenomena, only the expression of the relevant geographical features can be used to reveal the nature and laws of its scientific. Thus, people think of GIS technology applied to the field of visualization, through the graphical, symbolic, color of the geographical information expression to enhance and deepen the visual performance of the effect and function ${ }^{[5]}$. With the help of computer graphics, image processing technology and geography, the input, processing, query, analysis and forecasting data and results of geography information are displayed in graphical symbols, images and images ${ }^{6]}$. Through computer visualization, text, data, graphics, images, video and other information, the use of theory, methods and techniques for interactive processing, presented on the screen.

\subsection{Marine visualization characteristics based on GIS}

Compared with the traditional data visualization, GIS-based marine visualization features are mainly reflected in the following aspects.

\section{(1) Dynamic interactivity}

GIS-based marine visualization technology is dynamic interactive, it can provide users with flexible and effective means of control and use of information, while its database is constantly updated to ensure real-time, it's an important prerequisite for the spatial query and analysis function of GIS. When the system user carries on the analysis research to the marine data, may carry on the dynamic interaction with the computer very well, enables to take the initiative to find the phenomenon or the event which oneself needs, conforms to the reality.

(2) Diversification of information expression

Because of the large amount of data, the different format, the complexity of processing and other factors, based on GIS technology of marine visualization expression is no longer limited to basic data, text, but expanded into multimedia forms, such as graphics, Video and so on. Can be more colorful and complete and reasonable performance of information data, so as to multi-level, multiangle, multi-faceted performance of the characteristics of the geographical space environment, enriched the content and function of marine GIS visualization.

(3) Variety of information expression

Based on GIS technology of marine visualization can not only represent the spatial distribution of data and the relationship in the geographic chart, but also can reflect the development and change of the marine data through the description of time dimension and the visualization method. For example, by means of marine GIS visualization technology and time series to express particle 
current in a certain period of time within a large-scale flow, the process of change etc..

(4) Spatiality

Compared with the general visual expression, the fundamental difference is that the visualization based on GIS technology has more obvious spatial characteristics, which can reflect the space and scope of marine information, and can combine the marine information subject with the geographical space features to be more fit together.

\subsection{The process of visualization of marine data spatial information}

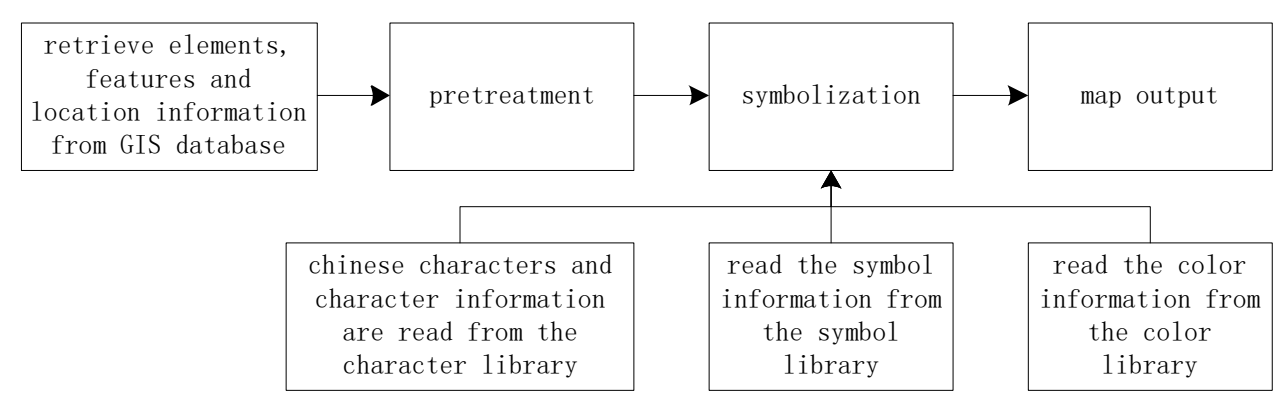

FIGURE 10. Visualization Design Process of Marine Data Spatial Information

As shown in Figure 10, this picture shows the marine data spatial information visualization process, from the following three aspects of design.

(1) Retrieval of GIS data from the geodatabase

In order to achieve the demand of visualization, a region is selected, the geographic object of attribute combination is selected by orthogonal analysis, the query is carried out in the GIS database, and then the results are classified, adjusted, combined and change according to their quality and data. According to the classification results in the above steps, classification coding is carried out to establish or switch the matching symbol base.

(2) Spatial data preprocessing

Because the current GIS spatial data sources in a wide range of data, the format is different and there is no uniform specification to build a visualization system, and from the numerous GIS database to read the right and appropriate data is a very difficult process. Therefore, in the use of these data, the need for further data on the type and format conversion, such as the amount of conversion, relational conversion and raster conversion.

\section{(3) Map symbolization}

At present, large and medium-sized GIS systems usually design symbol database and management symbols function, such as point query, line query and regional query, will refer to the actual demand design point symbols, line symbols and surface symbols. When the visualization system draws out the drawing, it will find the corresponding symbol from the symbol base and carry on the accurate localization and symbolization according to its spatial entity type.

For the marine visualization technology based on GIS can make people more convenient to study and observe the characteristics of marine phenomena and the spatial distribution of marine, in order to obtain new knowledge and laws of the marine. So the form of visualization of marine data will be used with the GIS system based on the characteristics of geospatial information.

\section{CONCLUSION}

Data explosion is a major challenge in the field of information science today. Not only is the amount of data needed to be processed is increasing, data is high-dimensional and multi-source, but also, more importantly, the dynamic and complex data acquisition. So it is very necessary to get from the massive data to which the law to make it into easy-to-understand graphics. Data visualization just solves this problem. So the data visualization for marine data, geographical data and other researches has a crucial role [7].

This paper studied the classification, comparison and methods of the visualization of expression 
of marine data, and the visualization of marine data spatial information based on GIS is studied, the characteristics of marine visualization based on GIS are proposed and to design the visualization of ocean data spatial information. The next step is to realize the visualization of marine data according to the design process, to provide the data support for the related marine scientific research departments, to construct the marine information system which integrates data management and visualization.

\section{REFERENCES}

[1] Liu Xiaofan, Deng Na. Depth discussion on visualization method of marine hydrological data based on GIS [J]. Science \& Technology Information, 2010(13):49-50.

[2] Tang Zesheng. Visualization in Scientific Computing [J]. China Economy \& Informatization, 1996(Z1):5-7.

[3] Yang Qiuqi. Research and Application of the Marine Environment Data Visualization [D]. North China University of Technology, 2016.

[4] Wang Xianghong. Research on Marine Environment Data Dynamic Visualization Based on 3D Virtual Earth[D]. Liaoning Technical University, 2013.

[5] Li Boyu. Web-based GIS Visualization System [D]. Zhejiang University, 2014.

[6] Zhang Feng. The Research of Representation on the topic geographic information in GIS [D]. Liaoning Technical University, 2009.

[7] Zhang Caizhang. Marine data visualization and application research [D]. Hangzhou Dianzi University, 2015. 